\title{
The Theory of Zero Incident Safety Management
}

\author{
Yin Wang*, Fletcher Griffis \\ Civil and Urban Engineering, New York University Tandon School of Engineering, New York, US
}

Email address:

yw620@nyu.edu (Yin Wang), griffis@nyu.edu (F. Griffis)

${ }^{*}$ Corresponding author

\section{To cite this article:}

Yin Wang, Fletcher Griffis. The Theory of Zero Incident Safety Management. Journal of Civil, Construction and Environmental Engineering. Vol. 3, No. 3, 2018, pp. 83-98. doi: 10.11648/j.jccee.20180303.15

Received: June 20, 2018; Accepted: July 7, 2018; Published: August 16, 2018

\begin{abstract}
Despite the dramatic improvements in construction safety made over the past few decades, the construction industry as a whole is still nowhere near reaching the Zero Injury goal, let alone the Zero Incident goal. The purpose of this paper is to provide an approach to continue improvements of safety performance in order to reach the Zero Incident goal. Since the concept of "Zero Injury" emerged in the 1990s, increasing numbers of owners and contractors have achieved the Zero Injury goal on construction projects. The key to these achievements is the Nine Zero Injury Principles established by Construction Industry Institute (CII). However, the research on which the Nine Zero Injury Principles relied is questionnaire survey rather than theoretical analysis. Without theoretical analysis supporting the research, the conclusions are often challenged, making the concept of Zero Injury difficult to promote throughout the industry. Thus, there exists the need to develop the theory of Zero Incident Safety Management (ZISM) and re-define the concept of "Zero Incident" to provide theoretical insight into the practical application of ZISM techniques and make Zero Incident more acceptable. Drawing on previous theories of construction accident causation and prevention, a new update accident causation theory was developed. This research then identified the management factors that can eliminate the corresponding accident causation factors. Developing the theory of ZISM can not only helps construction leaders deeply understand and accept the concept of Zero Incident, but also guide how to make Zero Incident come true. To facilitate the most rapid implementation of the Nine Principles, a ZISM standard benchmark tool with leading metrics was created. This tool can be easily used to assess the extent of implementation of safety program in construction projects.
\end{abstract}

Keywords: Construction Safety, Zero Incident, Zero Incident Safety Management (ZISM), Accident Causation Theory

\section{Introduction}

The "Zero Injury" concept was first described by CII in the fall of 1993 to show owners and contractors how to achieve zero accidents on construction projects. CII sought to convince management of the value of an effective safety program by researching and identifying techniques most successful in achieving zero accidents.

The Zero Injury philosophy is based on the belief that eliminating all worker injuries on projects is possible and is the only acceptable goal. "Zero Injury," including Zero Lost Time Injury and Zero OSHA Recordable Injury, defines a unique attitude on projects achieving the category of "safety excellence." This attitude appears as a zealous commitment by top management to the concept that Zero Injury is the only acceptable goal. The Zero Injury concept means that all serious injury to workers can be successfully prevented.

Emmit J. Nelson [1] offers his take on the potential scope or application of the Zero Injury concept:

"The essence of the Zero Injury concept is for a company or organization to adopt the notion that employee injury is not a desirable or an acceptable result of work execution. Thus, these devotees wish to experience Zero Injury for as long a period or for as many work hours as they can. They first strive for zero lost workday cases, then-when successful—for zero recordable, and then for zero first-aids."

The majority of construction companies have comprehensive safety programs, as reflected in their contractors' selection, training, inspections, enforcement, as well as efforts towards fostering a strong safety culture, and behavior-based safety plans aimed at decreasing the construction accidents incidence rate. The construction accident statistics from OSHA and the BLS 
from recent decades show that these companies have enjoyed significant improvements in their safety performance in the past decade. However, the industry is still nowhere near reaching the Zero-Injury goal, let alone the Zero Incident goal. The problem of not knowing how to continue improving safety performance to reach Zero Injury has been pervasive throughout the construction industry for a long time [2].

This paper targeted an approach known as Zero Incident Safety Management (ZISM) to achieve the Zero Incident goal. The essential questions of this paper are: what is the theory behind Zero Incident Safety Management and how can construction leaders use this theory to improve project safety performance to achieve the Zero Incident goal?

The challenge for this research is that safety management as part of the overall management process is affected by many factors, and it is difficult to identify and measure which factors are involved in developing the theory of ZISM. The main challenge lies in the difficulty in determining what to measure and how to effect improvements, particularly with respect to those intangible aspects of safety.

Therefore, this paper intends to provide theoretical insight into the practical use of ZISM within the field of construction safety. It will not only analyze the theory of ZISMincluding theory, process, methodology and tools - it will also develop a measurement tool to make the findings from this study easily applicable to construction projects. This would allow a strategic move towards a high performing and continuously improving safety culture, with the result of achieving and sustaining the goal of Zero Incident.

\section{Literature Review}

The first known construction contractor to exceed one million hours worked with zero OSHA recordable injuries was Zachry, a contractor based in San Antonio, Texas, while working on a Shell Chemical project near Baton Rouge, Louisiana in 1989. Since that time, increasing reports of zero recordable injuries were submitted. As a result of all CII attention the "one million" hour threshold became a commonly used measure of safety achievement that was beyond the norm and thus worthy of attention. According to the latest data, by 2015, 32 companies had reported a total of 92 occasions where projects had logged zero recordable injuries exceeding one million hours.

The National Academy of Construction (NAC) lists the names of known contractors (see Table 1) reported to have reached and exceeded the one million hour milestone, though a large number of them have exceeded two million hours and even three million hours worked with zero OSHA recordable injuries. In addition, two have reported exceeding four million hours recordable-injury free. [3]

Table 1. List of Contractors with Zero Injury over Million Hours.

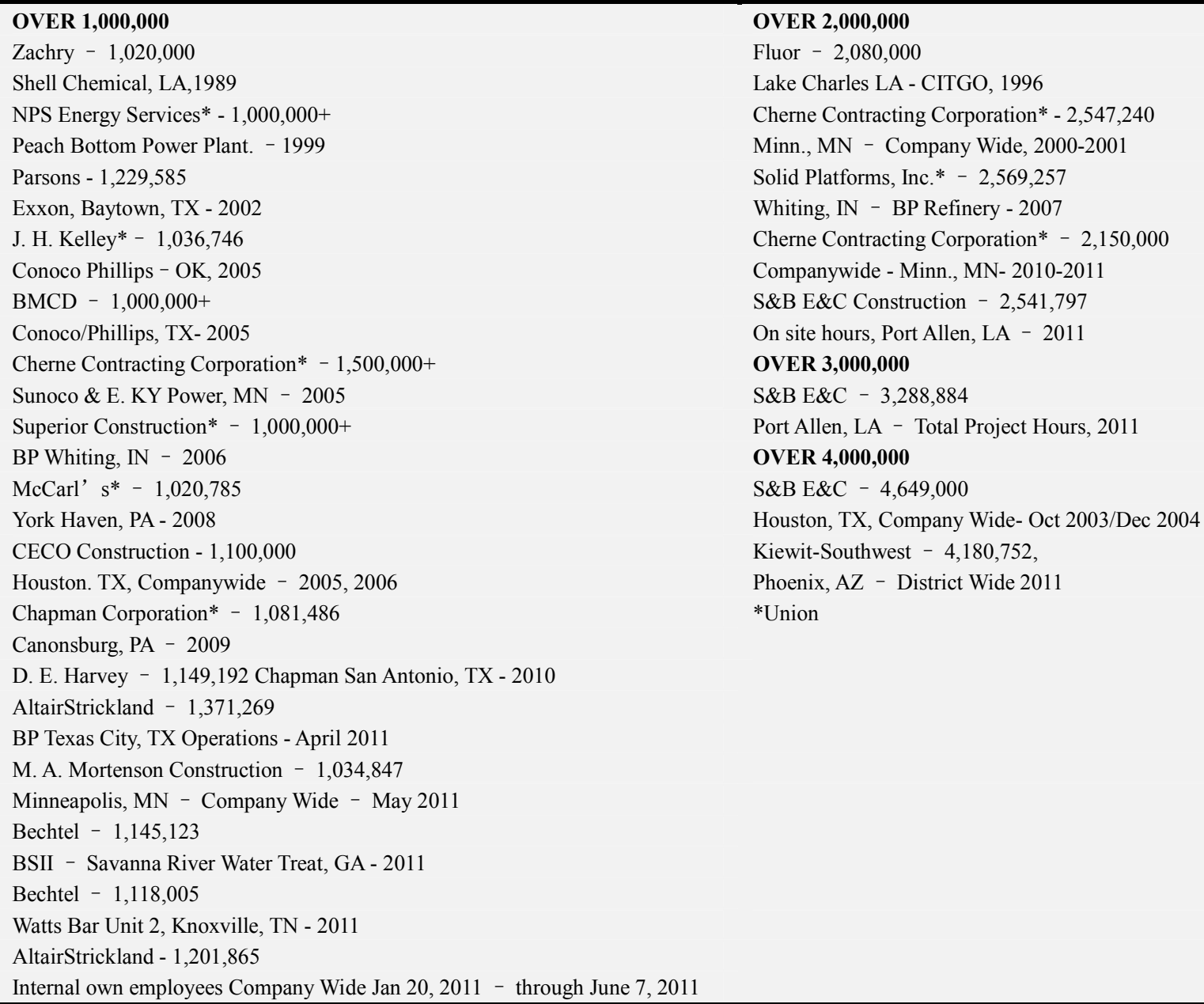


These remarkable achievements raise the questions of "how did they do that?" What kind of safety program does this company use to achieve such heights in the avoidance of employee injury? What are the key ingredients of a Zero Injury safety initiative?

The answers lie in the fact that these companies take full advantage of the Zero Accidents research done by the CII. This research details the major categories of safety leadership required to implement the highly successful "Zero Injury Safety Management Concept." Hundreds of other contractors across the USA have joined in successfully using this landmark research [3].

\subsection{Nine Principles}

The Nine Principles established by the CII research indicate how to attain their safety goals. The Zero Injury approach is becoming more widely accepted by both owners and contractors, both domestically and internationally.

The CII Report 32.1 "Zero Injury Techniques" in 1998, the subsequent studies in 2001 and 2002, and the follow-up research "Making Zero Injuries a Reality", defined Nine Principles to attain Zero Injury objective.

These nine CII Zero Injury Safety Management principles are as follows:

1. Demonstrated management commitment

2. Staffing for safety

3. Safety planning

4. Safety training and education

5. Worker participation and involvement

6. Recognition and rewards

7. Sub-contractor management

8. Accident/incident reporting and investigation

9. Drug and alcohol testing
The research demonstrated that companies utilizing the Zero Injury Nine Principles achieved better safety performance results than companies that did not utilize the techniques.

\subsection{Implementation of the Nine Principles}

Since publication of the report in 1993, several contractors using the Zero Injury safety principles continued to improve their safety performance, with some contractors experiencing zero recordable injuries [4]. Researchers named these practice policies "Zero Injuries Safety Management." These participating companies have had a large impact on the improvement of the RIR in the construction industry during the past two decades. In fact, as shown in Figure 1, the RIR statistics of CII member companies are an order of magnitude better than the construction industry ratings as a whole. Most, if not all, CII members have instituted the Zero Injuries Safety Management principles and philosophy. As a result, their total recordable injury rates are 10 times better than the industry average. Considering the actual labor-hours worked by the industry is about 12.6 billion man hours, the total number of injuries avoided should every construction company adopt the principles and philosophy of Zero Injury Safety Management would be in the neighborhood of 235,000 injuries per year (New Safety Initiative for the Construction Industry in the City of New York).

As a result of CII members' accomplishments, the Nine Principles are becoming more widely accepted by construction owners and contractors to attain Zero Injury goal. Some organizations, like the NAC and Zero Injury Institute (ZII), are actively advocating for the use the nine major categories of safety leadership cited above and for the more than 200 techniques found.

\section{RIR Industry vs. ClI Member}

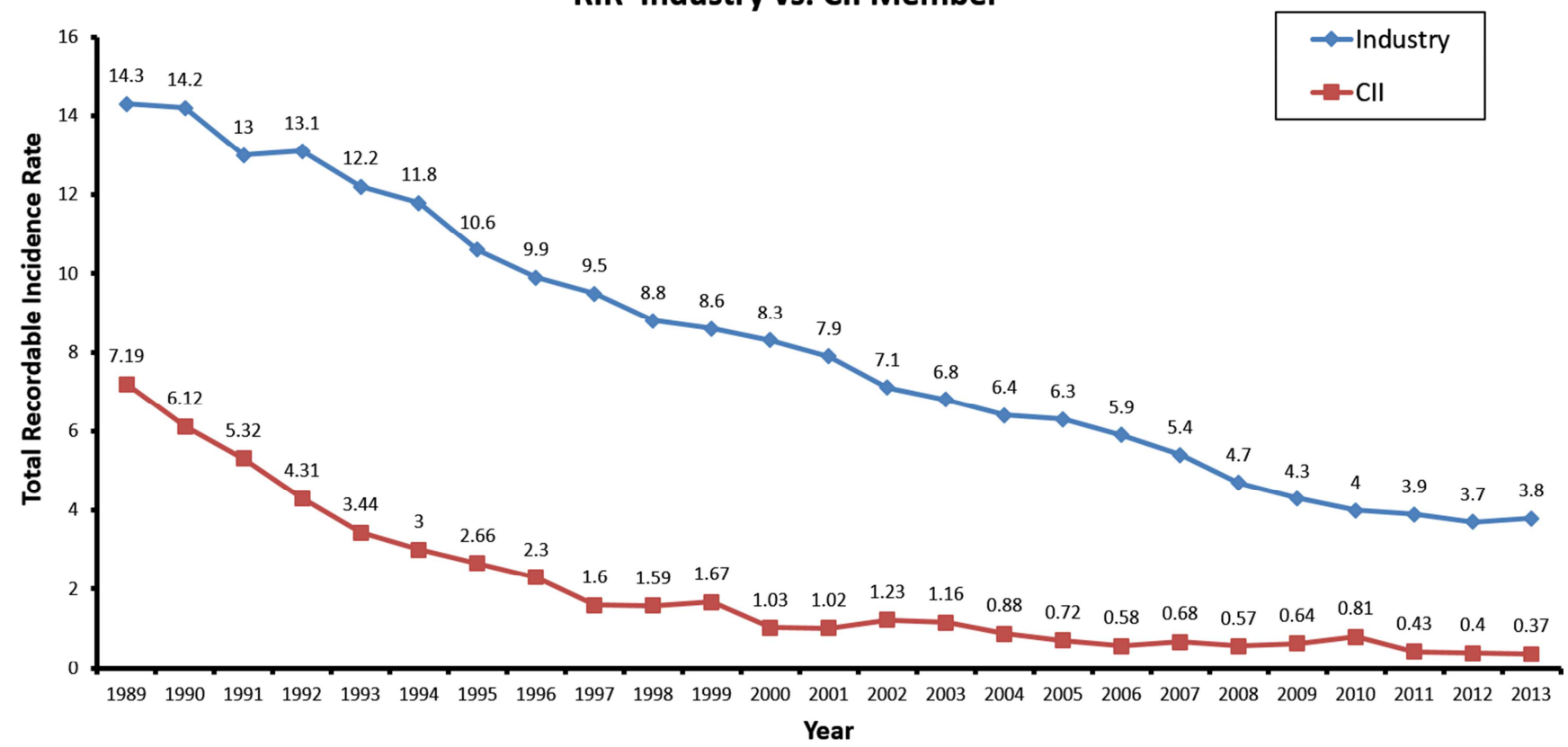

Figure 1. RIR of Industry and CII Member. 
It has been proved that successful safety programs consist of three primary parts: content, process and safety culture [1]. To achieve an effective safety culture, leaders must be engaged for the right reasons. If leaders engage in safety programs only because it is required by law, the result will likely be far less excellent than engagement by leaders who truly care for their employees.

Other research from ZII also concluded that safety success is heavily dependent on three factors: (a) Top leadership's routine communication of how zero at-risk behavior should be executed at work; (b) The use of research backed by leading safety indicators; and (c) The presence of employee safety knowledge at all levels in the organization. In short: "Safety performance correlates with safety knowledge!"

The Zero Injury philosophy is based on the belief that eliminating all worker injuries on projects for significant periods of time is possible. The first essential criterion required is the acceptance of the Zero Injury concepts by those in charge and the effective communication of this to the workers. Further, it is essential that owners and contractors devote resources for the development and implementation of the safety principles that provide the highest impact on achieving Zero Injury projects [2]. The construction industry needs to recognize that elimination of injuries is vital to the efficient execution of construction projects, and that productivity and safety are so intertwined in the workplace that spending time and energy on safety not only improves safety performance, but also avoids schedule delays and reduces costs [5].

\section{Problem Statement and Research Methodology}

\subsection{Problem Statement}

The Nine Principles from CII are becoming more widely accepted by construction owners and contractors who want to increase their safety performance. The CII research concluded that Nine Principles are the most effective approach to Zero Injury by collecting and analyzing project safety data. However, the research on which the Nine Zero Injury Principles is based relied on a questionnaire survey rather than theoretical analysis. Without theoretical analysis supporting this research, its conclusion is susceptible to challenges. In addition, the total of 38 interviews that CII conducted with safety management personnel on large construction projects in the U. S. was not convincing enough.

The concept of Zero Injury and the Nine Principles are often challenged due to lack of complete understanding of accident causation. There are many factors that can affect safety accidents or near-miss occurrences, a fact that makes it difficult to determine the cause of an accident. Research demonstrates that analysis of various factors is necessary to judge risk in a construction project [6]. Further, each construction site has its own conditions, location, number of workers, types of activities, and other unique characteristics. Without convincing evidence, it is difficult for owners and contractors to understand and accept the new concept completely. As a result, adoption of the Nine Principles can be a challenge for some companies. It's unfavorable for leaders to accept a new concept and spend a significant amount of money to make any changes with limited resources.

Consequently, there exists the need to conduct additional analytical research to develop the theory of ZISM by finding a method to determine the causes of accidents to 1) support the Nine Principles conclusions; and 2) develop an approach to help owners and contractors understand and accept the new concept. By doing this, more companies may be persuaded to adopt CII's Nine Principles.

\subsection{Research Methodology}

It is highly significant to propose a safety management methodology aimed at identifying those efforts needed to achieve continually greater periods of time worked without construction incidents. Consistent with the above goal, the objectives of the research include:

1. Developing the theory of ZISM for improving safety performance in the construction industry to achieve and sustain the Zero Incident goal.

2. Identifying the management factors involved in achieving the Zero Incident goal and developing leading metrics to measure these factors.

Social theory research is carried out in the tumultuous and ever-changing environment of functioning organizations. Construction projects, from the day they begin, are in a constant state of change. Research in the natural theory is typically carried out in a controlled laboratory setting that allows for the exclusion of extraneous factors; however, in this research, each construction project is unique and the question to be answered is: "Why can some contractors work a million hours without an injury while others cannot?" As an analytical research, the best way to solve the problem is based on combination of theory analysis and practical experiences. By summarizing and analyzing the related theories that have been approved and accepted in other industry and reviewing experiences from the companies that already successfully achieved the Zero Injury goal, the theory of ZISM and standard metrics will be developed. The general research work flow is shown in Figure 2.

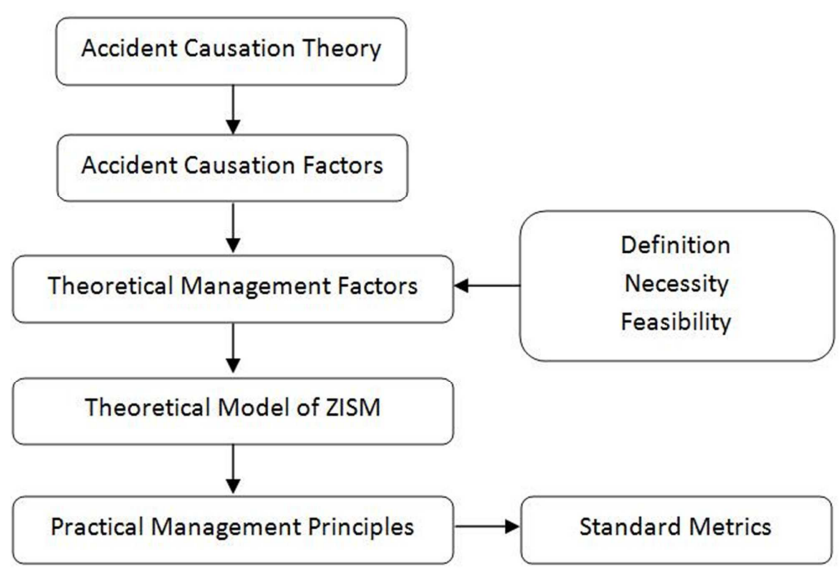

Figure 2. Research Workflow. 


\section{Developing Theory of ZISM}

The first phase of the research was to develop the theory of ZISM. The theory should include the underpinning theory and philosophy. To develop the underpinning theory and philosophy of Zero Incident Safety Management, it was necessary to identify the reasons why construction accidents happen. The process began with a thorough study of existing theories of accident causation and prevention. After summarizing and analyzing the conclusions of the existing theories, the most important reasons for why accidents happen were identified, and a new update to accident causation theory was developed.

After analyzing accident causation, the next step was to identify factors that might influence safety management. Based on this new theory, the management factors were concluded to eliminate the corresponding causes that lead to accident happening. The factors that involved in the theory of Zero Incident Safety Management can be defined. Then the theory of ZISM was developed. The theory is also a guide to provide direction for the following research to move closer to the Zero Incident goal.

\section{Creating Standard Metrics}

The main purpose of this phase is to create a ZISM standard benchmark tool with leading metrics, which can be easily used to assess the extent of implementation of safety programs in current projects and to compare them with safety programs of the successful projects achieved Zero Incident goal. The goal is to identify what companies that reach the Zero Injury goal do differently from companies that do not to move closer to the Zero Incident goal.

As discussed in literature review, the research conclusions found by CII can help companies achieve zero injuries. With the support of theory of ZISM, the Nine Principles could be deemed to the most effective guides for Zero Incident. This research also uses this conclusion and continues to discuss how to better implement the Nine Principles.

To facilitate the most rapid implementation of the Nine Principles, specific management actions should also be developed. James B. Porter developed thirteen actions in his recent research that would enable management to meet safety performance goals to be met sustain in the most cost effective and risk sensitive ways. The leading metrics in this research were created in accordance with the Nine Principles and the thirteen actions.

\section{Theories of Accident Causation}

It is extremely difficult to talk about construction safety management in the absence of an understanding of the causes of accidents. Before one can embark on effectively and efficiently improving safety on the project site, one must first understand the theory of accident causation and prevention.

Many attempts have been made to develop a prediction theory of accident causation, but so far none has been universally accepted. Researchers from different fields of theory and engineering have tried to develop a theory of accident causation that will help to identify, isolate and ultimately remove the factors that contribute to or cause accidents. In this article, a brief outline of various accident causation theories is presented.

1. The Domino Theory (Heinrich 1959)

Heinrich [7] was a pioneer in research on the conditions and circumstances that cause industrial accidents. He developed an accident causation theory known as the "domino theory."

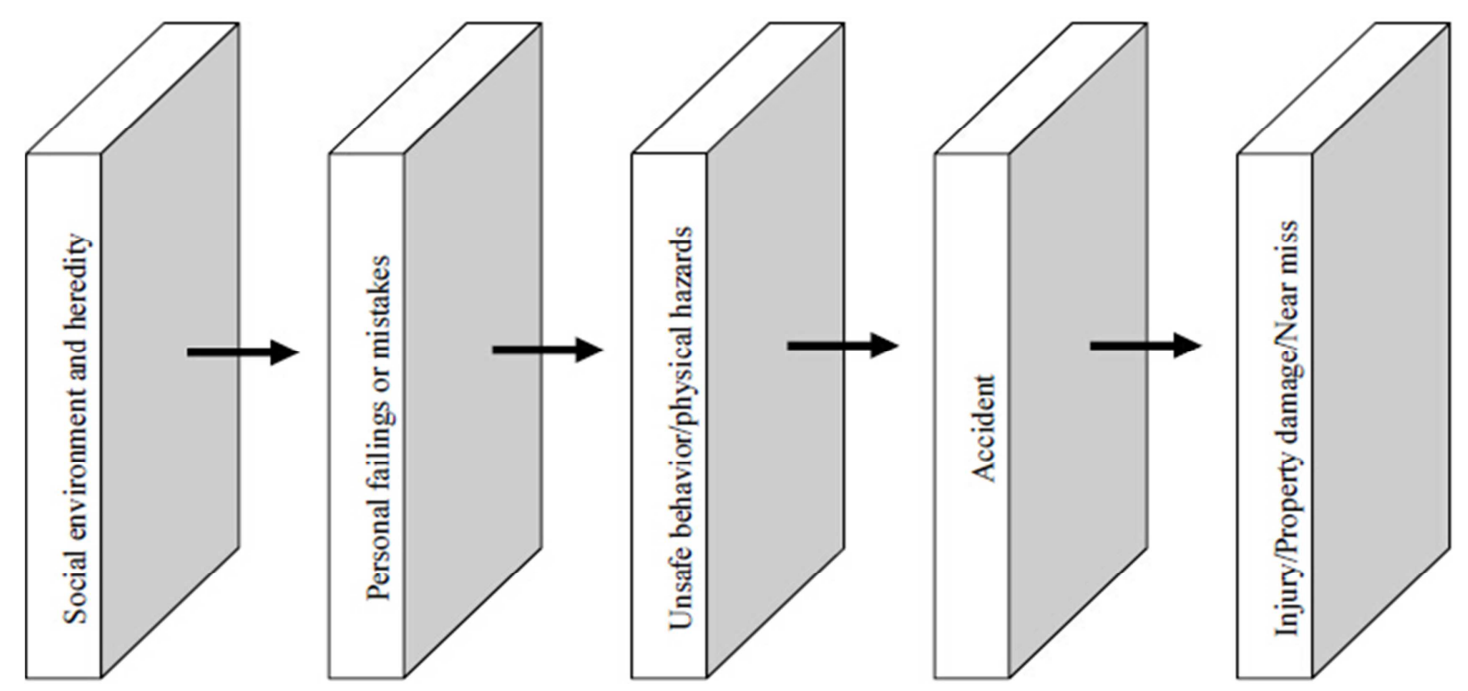

Figure 3. Heinrich's Domino Theory (Heinrich, 1959).

Heinrich's domino theory is comprised of five standing dominos that will fall one after the other if any domino falls (see Figure 3). An accident can be prevented only by removing one of the dominos preceding the accident. This interrupts the sequence and ensures that the accident does not happen.

The five sequential factors are as follows [8]:

i. Ancestry and social environment is the process of acquiring knowledge of customs and skills in the workplace. Lack of knowledge and skills required to perform tasks 
correctly and inappropriate social and environmental conditions will lead to the next domino: fault of person.

ii. Fault of person (carelessness) indicates negative features of a person's personality, although these unwanted characteristics might be acquired. Carelessness will lead to the next domino: unsafe act/conditions.

iii. Unsafe act or mechanical or physical condition includes the errors and technical failures which cause the next domino: the accident.

iv. Accidents are caused by unsafe acts or conditions and subsequently lead to injuries

$\mathrm{v}$. Injury is the consequences of the accident.

Heinrich's opinions were criticized for overemphasizing the immediate causes of accidents and neglecting an important aspect of accident causation and prevention: management practice [9]; however, Heinrich's research and work was the foundation for many other researchers.
Heinrich's domino theory has been modified and updated over the years with greater emphasis on management as an original cause of accidents. The management-based theories define management as responsible for causing accidents, and they attempt to recognize failures within the management system [10].

\section{Multiple Causation Model}

The Heinrich domino theory states that an accident is the result of a single cause. Petersen [11] developed a model based on management systems rather than individuals (See Figure 4). Petersen believed that there is more than one cause that contributes or leads to both the unsafe act, the unsafe condition, and, finally, the occurrence of an accident. Unlike in the simplified domino theory, there are causes and subcauses that contribute to an accident. Through identification of these multiple contributing causes of an accident, the unsafe acts and unsafe conditions should be prevented.

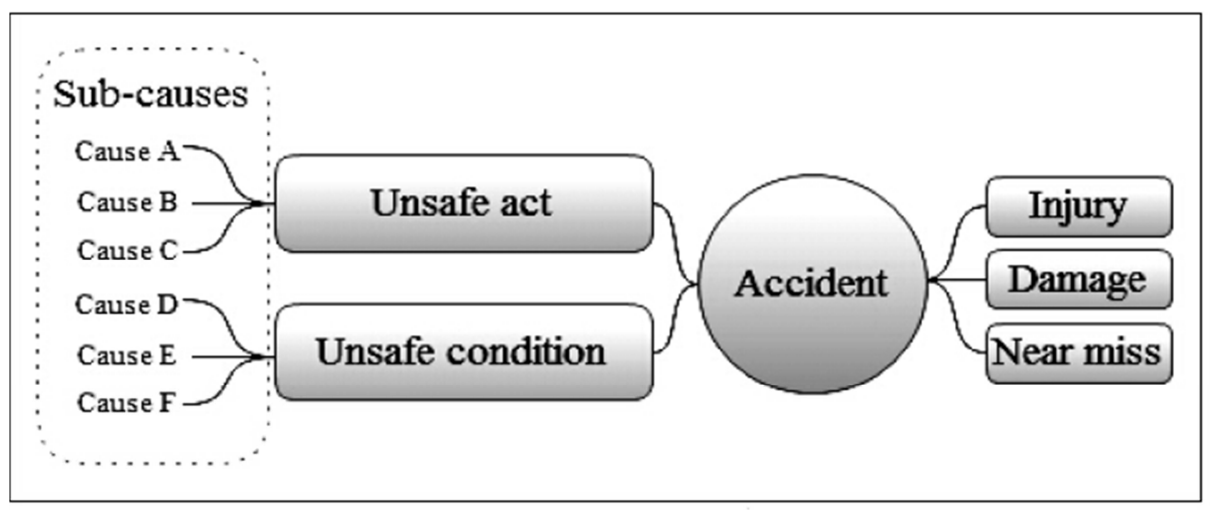

Figure 4. Multiple Causation Model (Petersen, 1971).

3. Updated Domino Sequence (Bird and Loftus, 1974)

Bird and Loftus (1974) also updated the domino theory to reflect the role of the management system in the domino- based model defined by Heinrich. The updated and modified domino model is illustrated in Figure 5.

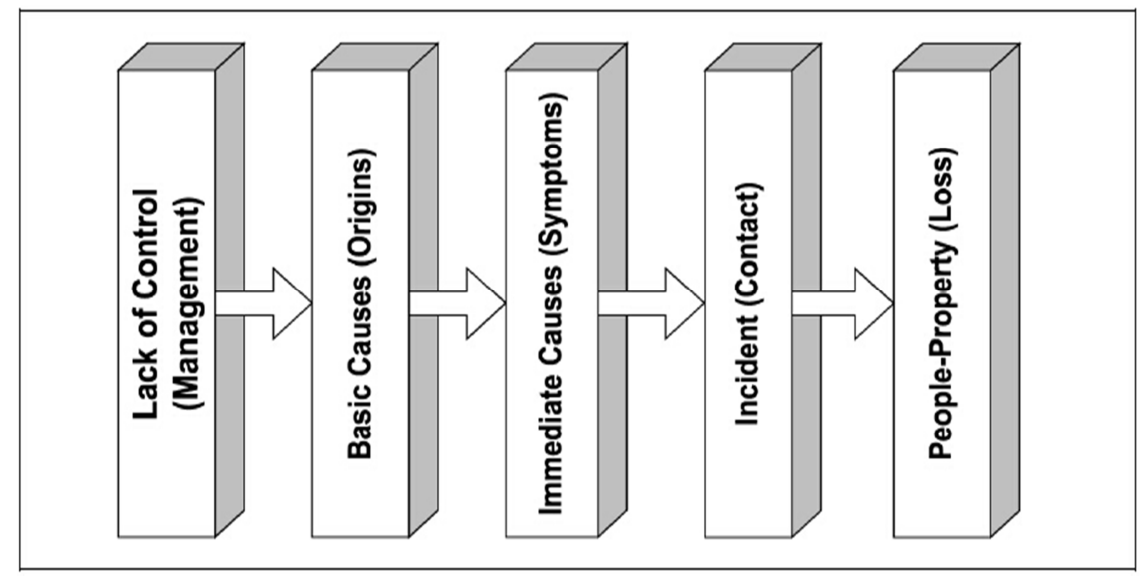

Figure 5. Updated Domino Sequence (Bird and Loftus, 1974).

i. Lack of control/management (inadequate program, inadequate program standard, inadequate compliance to standard)

ii. Basic causes/origins (basic causes: 1-personal factors, 2-job factors)

iii. Immediate causes/symptoms (sub-standard act and condition)

iv. Incident (contact with energy and substance)

v. Loss (property, people, process)

In this representation, the "Basic Causes" domino refers to factors such as a lack of motivation to work safely, or uncorrected hazards - factors over which management has a 
great deal of control. Each of the dominos in this sequence serves as an opportunity for intervening action, but the greatest potential for accident prevention is the first domino. When the domino theory is presented in this manner, management control is seen as the most important factor in the accident sequence [12].

\section{The Theory of Zero Incident Safety Management}

The analytic part of the study is the development of the theory of Zero Incident Safety Management to foster understanding and acceptance of the Zero Incident concept. The discussion of the theory of ZISM will include the following aspects: definition, necessity, feasibility, underpinning theory, factors, and models.

\subsection{Definition of Zero Incident}

The Zero Injury concept was first described by the Construction Industry Institute (CII) in the fall of 1993. Since then, as many companies have met the Zero Injury goal, the concept of Zero Injury has become more widely accepted by owners and contractors. Some specialist organizations, like the NAC and ZII, actively advocate the use the Nine Principles. All of these safety efforts use the concept of Zero Injury. The essence of Zero Injury is working with zero OSHA recordable injuries. According to OSHA General Recording Criteria-1904.7, an OSHA recordable case involves one or more of the following:

1. Death

2. Days away from work

3. Restricted work

4. Job transfer

5. Medical treatment (beyond first aid)

6. Loss of consciousness

7. Other serious or significant cases diagnosed by a physician or licensed health care professional (PLHCP)

8. Occupational injuries and illnesses that meets special recording criteria

However, injury is not the only serious consequence of accidents. Based on Heinrich's domino theory, Peterson's 1971 accident causation model concluded that, in addition to injury, property damage and near misses are also consequences of accidents (see Figure 4). The concept of Zero Injury does not include these additional consequences; however, from the perspective of safety management, it is important to take into account damage to property and near miss. Damage could directly lead to losses, and would probably affect the project schedule, even when there isn't a personal injury involved. The cost of the project could increase, as could insurance premiums.

A near miss, as defined by the National Safety Council, is an unplanned event that does not result in injury, illness, or damage to property, but has the potential to do so. Because a near miss does not incur loss in the form of injuries or property damage, and does not lead to substantial disruption, it has long been ignored in construction safety management research, and employees do not proactively report near miss incidents. Thus, many opportunities to prevent accidents are lost. Actually, a near miss is only a fortunate break in the chain of events that prevents an injury or property damage. History has shown repeatedly that most loss-producing events (accidents) are preceded by warnings or near accidents. Recognizing and reporting near miss incidents can make a major difference to the safety of workers.

Because near misses and property damage are significant consequences that should be considered, Zero Incident was used in this research instead of Zero Injury.

\subsection{Necessity of Zero Incident}

To better demonstrate the necessity of Zero Incident, it is first necessary to examine the difference between ZISM and other safety management. Any other goal of construction safety effort implies that accidents are expected and acceptable. In the very beginning of construction safety research, it was believed that accidents were a result of pure chance, and could happen to anyone at any time, though some people were more likely to suffer an accident than others. In the past, the prevailing view on construction safety accidents was that not all construction accidents were preventable. However, the Zero Incident philosophy is based on the belief that eliminating all worker accidents on projects is possible. The goal of all safety efforts is to increase safety performance and achieve "safety excellence." The difference between Zero Incident and other efforts lies in the former's claim that all accidents are preventable.

The essence of Zero Injury is working for a significant period of time with zero OSHA recordable injuries, while the essence of Zero Incident is working with zero OSHA recordable injuries, zero damage and Zero near misses. Once the essence of Zero Incident is clear, the necessity of Zero Incident can be approved by the method of "Proofs by Contradiction." If safety goals were not set as Zero Injury, employers, as leaders, send the message that some accidents are acceptable. As reported in recent research on safety culture, the leader's attitude and values have a significant impact on the whole company. Without Zero Injury, employees at all levels could have the wrong safety values and attitudes, which result in unsafe behaviors. What's more, the company safety culture would be influenced, and, as a result, more accidents would occur.

\subsection{Feasibility of Zero Incident}

All employers should have an absolute belief that it is possible to eliminate accidents. There are plenty of companies that have gone millions of man-hours without an injury.

The logic of Zero Incident is simple: if you can work safe on a task one time you can do it twice; if you can do it twice you can do it four times; if you can do it four times you can do it eight times; and so on and so forth. Zero Incident is not only a goal, but also a thought process that begins with the 
premise that if a worker can work one day without an injury, that worker can work each and every additional day injury free. The fact that an injury occurs does not mean it is acceptable to the organization [4]. Because Zero Incident is possible, accident prevention is no longer just a business proposition, but a moral obligation. If you can keep people from being injured, you have a moral obligation to do so.

\subsection{Underpinning Theory of Zero Incident Safety Management}

As discussed in Theories of Accident Causation, Heinrich (1959) was the pioneer in conducting research on the conditions and circumstances that caused industrial accidents. He developed an accident causation theory known as the Domino Theory.

Heinrich's research and work was foundational for many other researchers; however, his opinions were criticized for placing too much emphasis on the immediate causes of accidents while neglecting an important aspect of accident causation and prevention: management practice (Hosseinian \& Torghabeh, 2012).

Therefore, the Heinrich Domino Theory has been modified and updated over the years with greater emphasis placed on management as an original cause of accidents. Bird and Loftus developed another domino theory to reflect the role of management systems in the chain of what leads to accidents.

In Bird's representation, the "Basic Causes" domino refers to factors, such as a lack of motivation to work safely and other factors such as uncorrected hazards, over which management has a great deal of control. Each of the dominos in this sequence serves as an opportunity for intervening action, but the greatest potential for accident prevention is the first domino. When the Domino Theory is presented in this manner, management control is seen as the most important factor in the accident sequence [13].

By summarizing the two theories and interviews with safety expert, the new Wang's theory of accident causes and prevention was developed, shown in Figure 6.

The five important accidents causation factors are as following:

Environment and heredity: A person's undesirable characteristics such as recklessness, stubbornness, greed, and bad temper can be passed along through inheritance or developed from their environment. In other words, such traits can be caused by nature or nurture. Environment and heredity could be defined as people's undesirable traits that are affected by the environment and can contribute to faults of person.

Management: Management is classified as the underlying cause. Management controls the factors that prevent incidents from happening, including safety planning and organizing.

Personal factors: These factors include personal undesirable characteristics such as bad temper, inconsiderateness, ignorance, recklessness, and other personal problems that are not work-related, such as mental problems, illness, bad attitude, and lack of understanding, ability or motivation.

According to Heinrich, natural or environmental flaws in the worker's family or life cause these secondary personal defects, which are themselves contributors to unsafe acts, or and the existence of unsafe conditions.

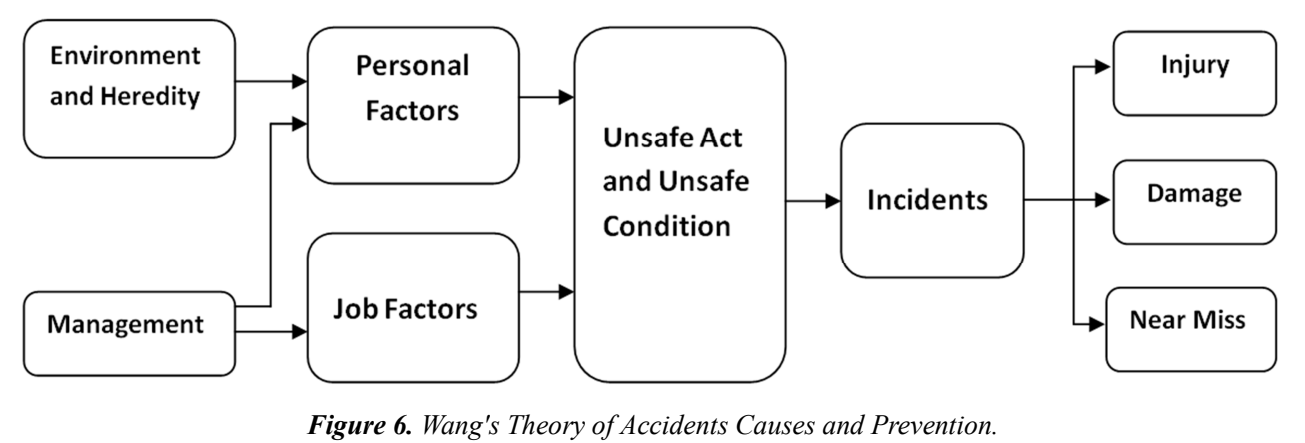

Job factors: These factors include all uncorrected hazards, such as acts of God, unsafe methods, job site conditions, unsafe equipment, inadequate work, normal or abnormal wear and tear, low-quality equipment, and bad design or maintenance.

Unsafe acts and unsafe conditions: These refer to the errors and technical failures that cause the accident and are the most significant factors that lead to accidents. Heinrich felt that unsafe acts and unsafe conditions were the central factor in preventing incidents, and the easiest causation factor to remedy, a process which he compared to removing one of the dominoes from the line.

All of the five causes were defined as the most important factors that result in construction accidents. Zero Incident
Safety Management should be developed by eliminating these causes. To achieve this objective, the safety management factors should be identified based on the new theory of accident causes and prevention.

\subsection{Factors Involved in Developing ZISM}

Zero Incident Safety Management is an approach that considers all of the factors that might influence safety, such as leadership, workers' behavior, and companies' cultures. Because it accounts for all of these factors, ZISM can function effectively in the dynamic, complex, and competitive conditions that construction projects face.

According to the new theory of accident causes and prevention, Zero Incident Safety Management should be 
contributed to eliminate the five causes.

1. Environment and heredity. To solve this problem, all leaders and employees have to understand the Zero Incident concept. Only people who understand and accept the concept deeply can change their safety values, and therefore their behavior. In addition, safety culture and safety training can also have an impact on this cause.

2. Management. Management depends on leadership. Employers' willingness to invest resources, time, and money in safety plays a significant role in safety management. Safety actions and the implementation of safety programs are also effected by management.

3. Personal factors. This is similar to environment and heredity. Safety culture, understanding of safety, safety actions, and the implementation of safety programs can change personal undesired characteristics and other problems.

4. Job factors. Uncorrected hazards, even an act of God, can be managed and prevented by proactive and strong leadership. The willingness to invest in effective safety actions and the implementation of safety programs can also help eliminate undesired job factors.

5. Unsafe acts and unsafe conditions. Unsafe errors and technical failures are the easiest causation factor to remedy. Strong leadership, proactive safety culture, specific safety actions, and implementation of effective safety program can solve this factor.

By summarizing the theoretical safety management factors that can eliminate the causation factor in preventing safety accidents incidents, the essential factors to achieve the ZISM is concluded as follows (see Figure 7):

1. Strong and active leadership.

2. The leader understands and accepts the Zero Incident concept.

3. Safety culture.

4. The owners and contractors are willing to invest in the implementation of the safety program.

5. The process of implementation of the effective safety program.

6. The effective safety actions that provide the highest impact on achieving Zero Incident.

The six management factors offer a theoretical guide that provides direction for where the efforts can be spent to achieve the Zero Incident goal. Among them, the understanding of Zero Incident and willingness to invest in safety are abstract psychological parts. Specific safety actions, implementation of safety programs, safety culture and leadership are all specific operational parts. The Nine Principles concluded by CII, as practical-level management factors, are included in the operational parts. The six theoretical management factors explain and support the Nine Principles found by CII.
Causation Factors

\section{Theoretical Management Factors}

(Involved in Theory of ZISM)
Practical Management

Factors

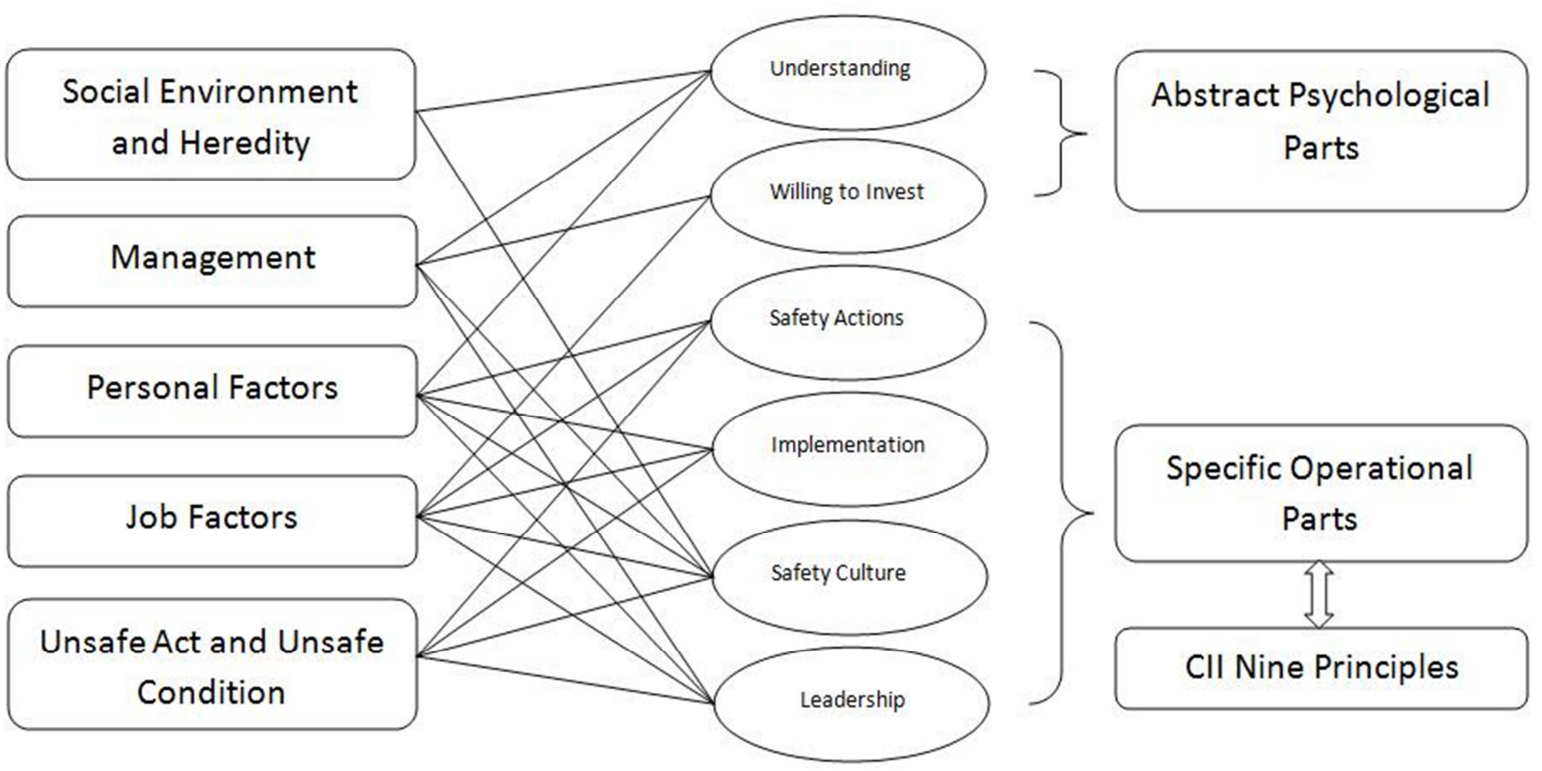

Figure 7. Factors Involved in the Theory of ZISM Development.

\subsection{Zero Incident Safety Management Model}

Because this is the first effort to develop a Zero Incident Safety Management model, the study is exploratory in nature. There are no previous studies that identify the ZISM factors and the nature of the relationships between the factors. Thus, there are no previous findings to confirm.

The current study developed such a model directed towards the Zero Incident goal by interviewing construction safety experts and analyzing the related materials. According to the identified safety management factors that involved in 
developing ZISM, the developed model was developed as shown in Figure 8.

1. Understanding and accepting the Zero Incident concept. The essential philosophy of Zero Incident is based on the belief that eliminating all worker incidents on projects is possible. Any other goal implies that incidents are expected and acceptable. Understanding the essence of Zero Incident helps leaders completely accept the Zero Incident goal, which is the first essential criterion required to achieve that goal. After they understand and accept Zero Incident, leaders will have the right reasons to truly care for their employees. Further, leaders can communicate the Zero Incident concept to their employees effectively to achieve an effective safety culture among the whole company. Finally, it is essential that the owners and contractors are willing to devote resources, money, and time to achieve the Zero Incident goal.

2. Willingness to invest. Willingness to invest in safety was defined in this research how much time, energy, resources, money, and others efforts an owner and contractor want to invest in construction safety. Only if leaders are willing to invest in safety will be able to achieve the Zero Incident goal and will show that they truly care about their employees. This is required to make strong leadership and active safety culture. This part will be further discussed in other paper.

3. Leadership. One important purpose of leadership is to create an organizational culture to guide the behavior of its members [14]. One of the primary duties of a leader is to create, develop, maintain and manage the culture of his or her organization. The creation of a desired organizational culture requires leadership to have a vision of the desired culture and to be active in communicating and selling that vision to the organization's members.

In a study titled Project Site Leadership Role in Improving Construction Safety, CII identified six leader behaviors that directly influence a safety culture in an organization and, ultimately, produce better safety performance. This research also concluded that developing a strong, positive safety culture requires active leadership that can influence values, attitudes, and beliefs about safety. Leadership behavior must reinforce the values, beliefs, and assumptions that constitute the desired safety culture in the project workforce and that affect worker behaviors [15].

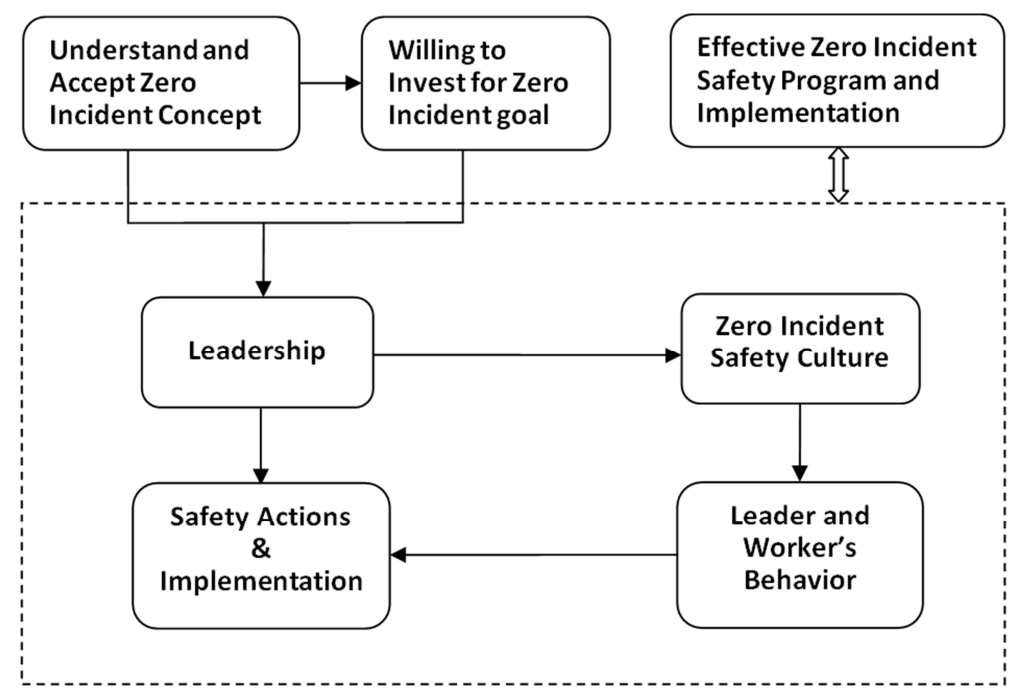

Figure 8. ZISM Model.

4. Zero Incident Safety Culture. A strong safety culture embraces the Zero Injury concept. An owner and contractor build a Zero Injury safety culture through strong leadership that pushes all employees at all levels to accept ownership of the safety performance objective of Zero Injury. Leadership also plays an essential role in its long-term success.

Existing research about safety culture showed that company's success in attaining a Zero Injury Safety Culture depends on a company's owners and senior leaders, who must instill, reinforce, and sustain the culture. Companies that meet this challenge with a relentless passion for Zero Injury have discovered that, regardless of the quality of safety systems, the number of safety meetings, the quality of training, the number of safety professionals or the number of processes required for a task, it is the strength of the leadership within a company that determines how close it can get to a constant zero [16].

5. Safety Program and Implementation. Safety programs can be broken down into hard content or soft content. Hard content is anything that falls under the category of statutory safety procedural and compliance techniques (e.g., all OSHA and DOT and other regulatory body stipulations applying to such things as scaffolding, rigging, welding, drilling, cleaning, and driving). Soft content is anything done for the employees (e.g., how they are treated, personal evaluations, safety recognition, 
safety participation and other employee-focused leading indicator safety initiatives). All specific safety actions and other safety measures about leadership and safety culture are included in safety program.

Implementation covers the "how" and "when" of a safety program. For the "how" detailed plans are prepared to ensure the quality of the safety program elements implemented. For the "when," an integrated and coordinated safety leading indicator implementation schedule is made and it is executed in a timely fashion.

After the ZISM model was developed, it was instrumental in understanding the Zero Incident concept and guiding the direction of the efforts spent on the Zero Incident goal. Based on this model, there are generally three parts need to focus on to achieve the Zero Incident goal: understand and accept the Zero Incident concept; have the willingness to invest in the goal; and develop an effective Zero Incident safety program. The main purpose of theory of ZISM is to help owners and contractors to understand and accept the Zero Incident concept.

\subsection{ZISM Model Validation}

According to the theory of ZISM developing process, validating the ZISM model is to validate the six theoretical management factors. For the specific operational parts, in other words, the Nine principles have already been approved by CII research. The CII Report 32.1 "Zero Injury Techniques" in 1998, the subsequent studies in 2001 and 2002, and the follow-up research "Making Zero Injuries a Reality" have concluded that Nine Principles are the most effective approach to Zero Injury by collecting and analyzing project safety data. For the Willingness to Invest in safety part, the other paper "The Approach to Increase Willingness to Invest in Safety (WTI) for Zero Incident'has the details to demonstrate the definition of Willingness to Invest in safety and how it works to increase safety performance.

\subsection{Standard Metrics}

While the idea of Zero Incident is new, not much research has been done to develop a strategic safety management approach for the construction industry that would allow for continuous measurement and improvement of factors determining the safety performance of a construction organization. To be instrumental in reaching and sustaining the goal of zero accidents, the current study delved into developing such a standard metrics directed towards the goal of implementation of safety program.

The Nine Principles concluded by CII offer the most effective guide. In addition, James B. Porter developed thirteen actions needed to enable safety performance goals to be met and sustained in the most business effective, risk sensitive ways. In this research, to facilitate the most rapid implementation of the Nine Principles and to develop a better safety program, both CII and Porter's conclusions were combined. The management actions were developed first, and, based on these actions, the leading metrics were created.

The explanation of the Nine Principles, management points, the thirteen actions and the metrics are shown in Table A1.

The metrics were composed of two parts: a checklist and a scorecard. To standardize the value of each metric, they were quantified as fixed scores. Then the standard benchmark can be used to evaluate any other construction projects and compare their safety management with Zero Incident Safety Management. The gaps in the safety action process and missing elements in safety management will be found and leaders will be given the opportunity to retrofit these if needed, so that they can continue progressing to the Zero Incident goal.

\section{Conclusion}

This paper mainly developed an approach, Zero Incident Safety Management (ZISM), to answer the question "How can construction leaders employ the theory of Zero Incident Safety Management to improve safety performance so as to achieve the goal of Zero Incident?"

To answer the question, the research generally had two objectives. Phase 1 of the research was to develop the theory of ZISM. Based on the literature review and current development situation of the construction safety, the new theory model of accident causes and prevention was developed. Then the safety management factors were identified and the ZISM model was created.

The theory of ZISM not only helps owners and contractors to deeply understand and accept the Zero Incident concept, but also can serve as a theoretical guide to provide direction for continuing increasing safety performance to achieve the Zero Incident goal. Based on this model, there are general three parts need to focus to achieve Zero Incident goal: understanding and accepting the Zero Incident concept, having the willingness to invest in the Zero Incident goal, and developing an effective Zero Incident safety program. The theory of ZISM includes the motivation, necessity, feasibility and the underpinning theory. The purpose developing this theory was to help owners and contractors understand and accept the Zero Incident concept.

In phase 2 of this research, based on the Nine Principles concluded by CII and thirteen actions developed by James B. Porter, the standard benchmark with leading metrics was created to facilitate the most rapid implementation of the Nine Principles to develop a better safety program.

However, the developed standard benchmark needs to be further validated by assessing the safety management in the real projects. The sample should include the projects that successfully achieved Zero Incident goal and target projects. By assessing the safety management of the projects, the collected feedback will be used to continue adjusting the leading metrics and the standard benchmark.

Eventually, the standard benchmark can be used to evaluate any other construction projects and compare those projects with Zero Injury safety management. The gap of safety techniques, process and safety management missing will be found and give leaders the opportunity to retrofit if needed, to continue progressing towards the Zero Incident goal. 


\section{Appendix}

Table A1. ZISM Standard Metrics.

\begin{tabular}{l}
\hline Category Definition, Explanation and Management Points \\
cheans a 1-5 quality rating scale, that might have descriptors as follows. 1 = Poor, \\
1. Demonstrated management commitment \\
1. This "demonstration" must begin at the very top of an organization; \\
2. The term "commitment" is defined as a "personal devotion to doing \\
whatever it takes to prevent an injury." \\
3. Senior management should communicate directly with field \\
personnel to demonstrate their concern over safety on the job and \\
their commitment to zero incident safety management.
\end{tabular}

2. Staffing for safety

1. Skilled safety staff must be provided with safety coaching, orientation, training, compliance oversight and safety inspections and audits.

2. Providing skilled safety staff in appropriate numbers to insure that all these needs are furnished at the appropriate time.

3. "Line management leadership" in implementing the zero injury safety technology. One should use care not to label the head of the safety support function as "Safety Manager", but Safety Director, Safety Representative, Safety Staff, and Safety Specialist.

3. Pre-project and pre-task planning

1. This planning is to be done in three distinct project phases: 1. PreProject Safety Planning, 2. Pre-job Safety Planning and 3. Pre-task Safety Planning.

2. All affected personnel are to be in attendance; For Pre-project and Pre-job safety planning, Top Project Leaders (both Engineering and Operations), Purchasing Executive, Project Safety Director, Project Engineers, Project Schedulers, Project Superintendents and Craft Superintendents. These meetings are called by and chaired by Project Leadership. Pre-task planning is accomplished by the Craft Foreman and crew so the crew is all present when each pre-task plan is made.

3. Pre-Project Safety Plan

4. Safety planning begins in the project planning stages. All parties to be engaged in the construction of a project should gather and spend significant time discussing the various phases of the project and what safety considerations should be observed during each phase. This should include site clearing, excavation, underground work, foundations, steel erection, equipment lifting and setting, piping installations, electrical, insulation and start-up. Each of these phases has distinct safety considerations that if planned and provided will enhance protection of the workforce from undue hazards. Then the plan is executed in detail by the project superintendents.

5. Pre-Job Safety Plan

6. This planning goes into more detail than safety planning accomplished in the phases mentioned above, heavy lifts included
Leading Indicators

Metrics

2 = Fair; 3 = Good; 4 = Very good; $5=$ Excellent. $\square$ means Yes or No

a) Are the top managers, upper level involved in worker safety, such as training, inspection, orientation, and accident and incident investigation?

b) The top managers actively supply the financial and human resources required for implementation of the zero injury safety management techniques.

c) At lower management levels, safety staff faithfully using those resources to effectively implement the various techniques.

d) Senior manager give feedback to field safety staff about their safety job periodically. (Like award superintendent for no safety incident during the period.)

e) Safety leaders have an instilled belief that their leadership is what prevents accidents.

f) The frequency of talking to workers about safety

a) Safety personnel are provided in every safety activities.

b) The ratio of workers to safety personnel changes depending on the timing of the need for these services.

c) The safety personnel involve in regular management meetings.

d) Set line management to insure all work has a safety plan and is executed according to accepted safe work execution practices.

a) The site-specific safety program begins in the project planning stages;

b) Senior leadership and all parties are intimately involved in pre-project planning;

c) Pre-Project plan discuss the various phases of project and what safety considerations should be observed during each phase;

d) All the pre-planning on safety is executed in detail by the superintendents.

e) All affected parties again go over the steps to be taken and safety precautions highlighted to insure each aspect of phase execution and safety oversight is adequately covered;

f) Pre-task planning and job hazard analysis is undertaken before each new serious task is started;

g) Foremen and crew members are involved to plan the details of each specific task;

h) Pre-task planning is done using a Task Planning Form to record the details with emphasis on safety and each member of the crew signs.

i) A new crew meeting is convened to reassess the plan if any changes happens, and resign.

j) For shift's work, a new Pre-task plan is made by each crew at the beginning of each shift. The pre-task plans should be reviewed by the General Foreman and/or 


\begin{tabular}{l}
\hline Category \\
\hline Definition, Explanation and Management Points \\
It is timed to slightly precede the beginning of each phase. In these \\
meetings all affected parties again go over the steps to be taken to \\
accomplish the job and safety precautions highlighted and \\
assignments made to insure each aspect of phase execution and \\
safety oversight is adequately covered. \\
7. Pre-Task Safety Plan \\
8. Prior to each task, foremen and crew devote the time necessary to \\
plan properly the details of each specific task and create a task \\
specific safe execution plan. This is typically craft-specific \\
planning but at times can involve multi-craft operations. The \\
foreman or a selected craft volunteer will lead the safety planning \\
discussion, outlining each step in the construction sequence, and \\
highlighting the safety considerations at each step. This planning is \\
done using a Task Planning Form to record the details with \\
emphasis on safety. Each member of the crew then signs the plan. \\
Should any change be thought necessary then a new crew meeting \\
is convened to reassess the plan, make any changes and then re-sign \\
the plan. This procedure is followed at the outset of each new task. \\
9. A given crew may prepare multiple Pre-task plans during the course \\
of a shift's work. As a minimum on repetitive or continuing work a \\
new Pre-task plan is made by each crew at the beginning of each \\
shift. The pre-task plans should be reviewed by the General \\
Foreman and/or Superintendent during each shift to insure \\
completeness, clarity, and quality. \\
4. Safety education: orientation and specialization training
\end{tabular}

1. The formal safety orientation is given all employees (new employees before they report to their foremen). It is essential that both project safety leadership and line management take part.

2. Each and every orientation session should never be simply videos alone, should include a presentation by an appointed representative of top project line management who clearly informs the attendees of leadership's commitment to them as individuals and to an injury free work place and personally ask for each employee's commitment to avoid all at-risk behavior.

3. The general contractors assumed the primary responsibility for orientation training of the subcontractor employees in addition to training their own employees.

4. Specialized training in pre-task planning and the safe usage of any unique tools (not commonly used) along with the appropriate use of personal protective equipment.

5. Safety training is a line item in the budget. From CEO's to the line workers provide resources for the safety education.

6. Comprehension testing was conducted to insure employee comprehension of the orientation details and understanding of zero injury is real and not just "safety talk."

7. Additional safety training should be provided in specific project in advance of need, at least four hours safety training per month. The frequency of training most prevalent was weekly with weekly supervisor training being a strong component.

8. Weekly toolbox is used to cover topics such as safety rules, hazards, corrective actions, accident prevention, a review of recorded injuries and near misses, and any major hazards that might be presented by upcoming project activities.

9. Rather than adhering to conducting these meetings on a rigidly set day of the week, conducting toolbox meetings when there is a greater need to communicate specific safety information.

Leading Indicators $\quad$ Metrics

Superintendent during each shift.

a) A formal safety orientation should be given to all employees.

b) Safety personnel and line manager participate in orientation.

c) The top manager clearly informs to employees leadership's commitment.

d) The general contractors assume the responsibility for orientation training of the subcontractor employees.

e) The amount of hours that safety training is given to workers beyond the orientation;

f) Assess training needs and develop and conduct additional programs tailored to the specific needs of individual groups.

g) All the training are held during regular working hours so that workers are being paid during training;

h) The frequency of safety training.

i) The weekly toolbox meetings cover safety rules, hazards, corrective actions, accident prevention, review of recorded injuries, near misses and any hazards that might be presented by coming activities.

j) Integrate safety messaging into current meetings and communications

k) Formal safety newsletters and bulletins are established.

1) The timing of toolbox meetings vary the day each week when needed. 


\begin{tabular}{l}
\hline Category Definition, Explanation and Management Points \\
5. Worker involvement \\
1. Involvement of the employees in participating in the setting of \\
safety objectives and goals thus enabling the employees to have a \\
voice in what needs to be done and how to do it. This involvement \\
necessarily comes after the employees have been given in-depth \\
education on the research proven safety element content of a zero \\
incident safety initiative. \\
2. Train the crafts who volunteer or supervisors to observe behavior of \\
workers to implement Behavior Safety. \\
3. The feedback from observations is confidential. \\
4. The observation data is tabulated as anonymous and reported back to all \\
employees weekly/monthly as a measure of "safe behavior." \\
5. Uses independent persons outside the project leadership to perform \\
employee safety perception surveys. Survey questions should be \\
based on the CII research best practices and focused on the quality \\
of the zero injury safety technique implementation. Insure "all" \\
project personnel are invited to participate. All input is to be on an \\
anonymous basis. \\
6. After the survey is conducted the data are tabulated and analyzed \\
focusing on finding opportunity for improvement and any remedial \\
immediate action can begin immediately
\end{tabular}

1. Performance evaluations of all employees should include one on one discussion of their individual safety performance. Ninety percent of the time leader discussions with employees are opportunities to praise individual safe behavior and also critically important $(10 \%)$ opportunities to conduct safety coaching of employees in need of improved individual performance. These discussions should be held at least annually.

2. Once zero injury performance is achieved group appreciation and recognition events should be held at least on a monthly interval.

3. Other safety milestone commemorating gifts and awards are in order however the value of these recognition items should not exceed a nominal dollar value.

4. Redesign your safety bonus/incentive system to avoid a focus only the outcome.

5. "Praise" then is a powerful employee motivational tool.

7. Subcontractor management

1. Careful contractor screening is done by the owner and/or the prime contractor at the proposal stages of a project to insure all contractors offering bids are indeed committed to the CII zero injury research fundamentals.

2. The screening process used to select eligible contractors should insure each bidder has successfully implemented on recent projects the CII zero injury safety techniques. Careful scrutiny of each contractor's approach to the utilization of the zero injury research should be conducted including interviews with the top contractor leaders that are being proposed to head up the project.

3. Contractors entering the project execution process some months into the project schedule should also be similarly screened. Should a contractor be found to be in violation of safety commitments given, the CII research suggests that the offending contractor/s be sanctioned until the violation/s is/are corrected.

4. Design and implement a Contractor Safety Management Process. 8. Accident and incident investigation

1. Prompt investigation of all accidents, incidents, injuries and near misses with appropriate feedback to all Employees.
Leading Indicators

a) Workers are involved in Safety committees;

b) Formal behavior-based safety program is developed;

c) Certain crafts or supervisors are trained to observe worker safety behavior;

d) Provide confidential feedback about their observations;

e) The observation data is tabulated as anonymous and reported back to all employees weekly/monthly as a measure of "safe behavior.

f) Uses independent persons to perform employee safety perception surveys.

g) Workers' overall feelings of safety;

h) Worker comfort levels around bringing up safety concerns to someone else's attention;

i) Employees' perception of company's support if they refused to work in an unsafe environment.

a) Performance evaluation of all employees is developed by one on one discussion of their individual safety performance;

b) Appreciation and recognition events are hosted;

c) Rewards system for safety workers;

d) The frequency of the recognition events with rewards.

e) Use the leading indicators to redesign the safety incentive system to focus more on process.

f) Praise employees enough.

a) Subcontractor screening is done by the owner and contractor at the proposal stage of project;

b) Check each subcontractor has successfully implemented the nine techniques on recent projects;

c) The offending subcontractors in violation of safety commitments is sanctioned until the violations are corrected.

d) Contractor safety management process is developed.

a) All incidents should be promptly investigated; b) Root cause analysis should be done to modify safety 


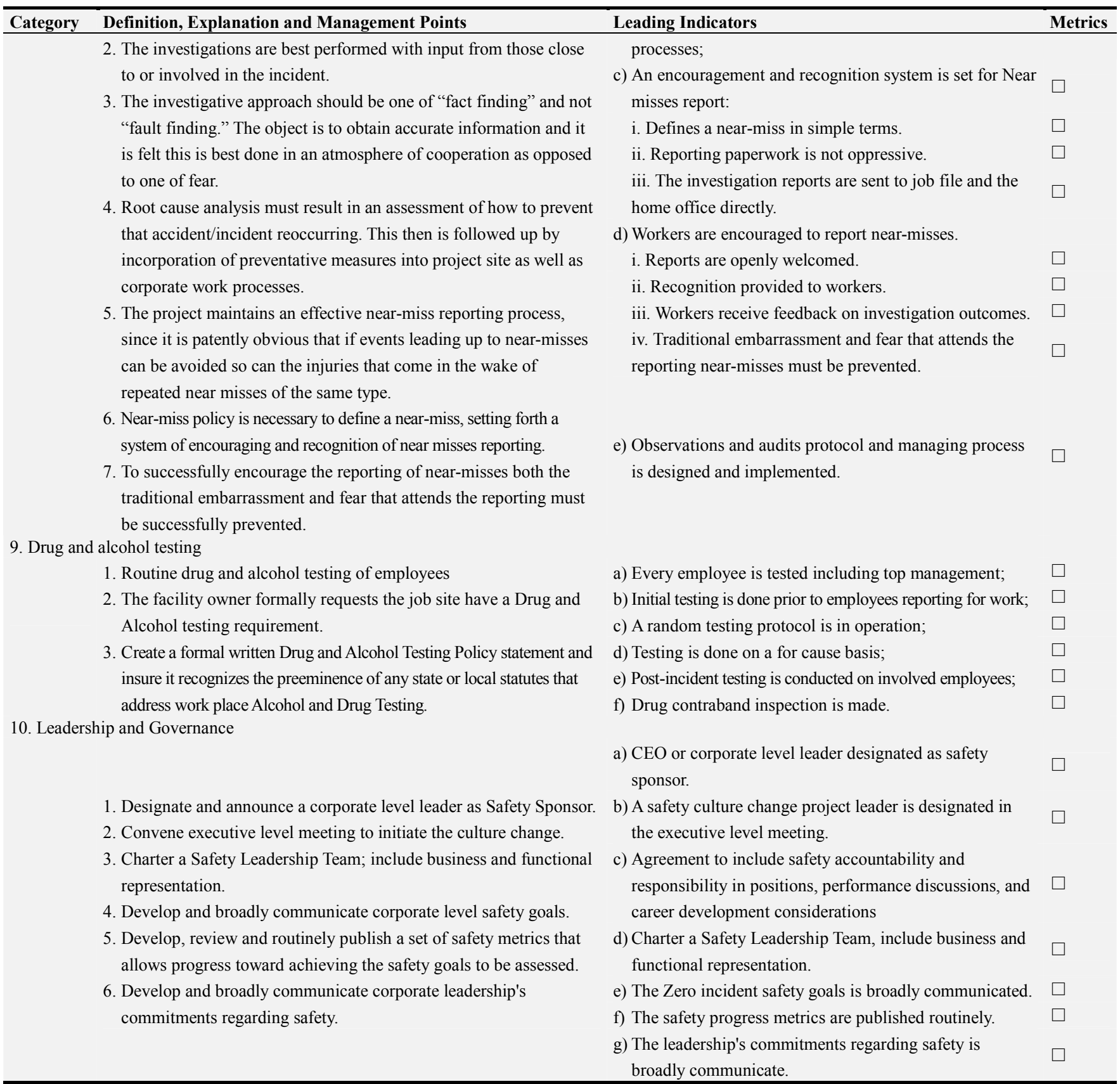

\section{References}

[1] E. J. Nelson, "Owners Reap Benefits from Zero Injury," in Collected Papers from the InterConstruct 2003, Stratfordupon-Avon, 2003.

[2] CII, "CII. Zero Injury Economics," CII Publication 32-2, September 1993; CII, "Zero Injury Techniques," CII Publications and Events Center, 1993.

[3] The National Academy of Construction, "The NAC SAFETY White Papers May 19, 2005 thru December 1, 2015," Construction Industry Institute, Austin, TX, 2015.

[4] M. Binsfeld, "Zero Injury Program," April 2010. [Online]. Available: http://www.hilmersonservices.com/safety/consulting/zero- injury-program

[5] R. U. Farooqui, Achieving Zero Accidents - A Strategic Framework for Continuous Safety Improvement in the Construction Industry, FIU Electronic Theses and Dissertation, 2011.

[6] J. A. Gambatese, "Safety emphasis in university engineering and construction programs," 2009.

[7] Heinrich, H. W. (1959). Industrial accident prevention : a scientific approach (4th ed.). New york: McGraw-Hill.

[8] G. E. K. a. H. R. Taylor, Enhancing Occupational Safety and Health, Elsevier Butterworth-Heinemann, 2004.

[9] S. S. Hosseinian and Z. J. Torghabeh, "Major theories of Construction Accident Causation Models: A Literature Review," International Journal of Advances in Engineering \& Technology, vol. IV, no. 2, pp. 53-66, September 2012. 
[10] R. Liska, D. Goodloe and R. Sen, Zero Accident Techniques, Construction Industry Institute, University of Texas at Austin, 1993.

[11] D. Petersen, Techniques of Safety Management, New York: McGraw-Hill, 1971.

[12] A. Raouf, "Theory of Accident Causes," in The ILO Encyclopaedia of Occupational Health and Safety, 4th Edition ed., vol. II, Geneva, ILO- International Labour Organization, 1998, pp. Part VIII-56. 6.

[13] J. H. W. William A. Stanton, "Conceptual Framework for Computer-Based, Construction Safety Control," Journal of Construction Engineering and Management, vol. 116, no. 3, September 1990.
[14] M. Rosenberg, "Ways Leadership Affects Culture and Culture Affects Leadership.," 24 July 2008. [Online]. Available: http://www.humanresourcesiq.com/hr-talentmanagement/columns/ways-leadership-affects-culture-andculture-affect

[15] CII, "Project Site Leadership Role in Improving Construction Safety," January 2012.

[16] M. Plews, "Safety Culture in Construction," 2 May 2012. [Online]. Available: http://jcoregon.com/news/2012/05/02/safety-culture-inconstruction. 